\title{
El uso de la rúbrica para evaluar tareas en entornos virtuales de aprendizaje: un caso aplicado a estudiantes avanzados de la carrera de economía

\author{
Using rubrics as an evaluation tool: a case in an advanced course of \\ undergraduates in economics
}

\author{
Irene Centurión César, María Cecilia Lara Martínez, Carolina Román Ramos \\ Departamento de Economía, Facultad de Ciencias Económicas y de Administración \\ Universidad de la República \\ Av. Gonzalo Ramírez 1926, Montevideo, Uruguay \\ irene.centurion@ccee.edu.uy clara@iecon.ccee.edu.uy croman@iecon.ccee.edu.uy
}

\begin{abstract}
Resumen
En este artículo se presenta la experiencia del uso de la rúbrica para evaluar tareas en entornos virtuales de aprendizaje incorporada a Economía de América Latina, asignatura avanzada de la carrera de la Licenciatura en Economía de la Universidad de la República, Uruguay. Se trata de una innovación docente aplicada este año a raíz del contexto de la pandemia, que implicó el diseño e implementación de una nueva herramienta para la evaluación del aprendizaje. Los resultados de su aplicación fueron muy positivos, tanto para el objetivo de evaluar los contenidos de la asignatura y el proceso de aprendizaje de los estudiantes, como para la corrección por parte de los docentes, y la devolución a los estudiantes. Asimismo, la opinión de los estudiantes se mostró muy favorable en relación con su uso.
\end{abstract}

Palabras clave: Rúbricas, Evaluación de aprendizajes, Enseñanza en economía.

\begin{abstract}
In this article we present the experience of the rubric to evaluate the students' performance on tasks in moodle in an advanced course of Latin America Economies as part of the Degree in Economics from Universidad de la República, Uruguay. This is a teaching innovation applied this year due to the context of the pandemic, which involved the design and implementation of a new tool for the evaluation of learning. The results of its application were very positive, both for the purpose of evaluating the contents of the subject and the students' learning process, as well as for the assessment by the teachers, and the feedback to the students. Likewise, the students' opinion was very favorable regarding its use.
\end{abstract}

Key words: Rubric, Learning evaluation, Teaching in economics.

\section{Introducción}

El presente artículo presenta la experiencia de una innovación docente en la evaluación de los contenidos teóricos de un curso avanzado de la carrera de la Licenciatura en Economía de la Facultad de Ciencias Económicas y de Administración (FCEA) de la Universidad de la República, Uruguay (UDELAR).

La UDELAR, cuenta con un Entorno Virtual de Aprendizaje (EVA) desde 2011, sin embargo, la modalidad no presencial no se había expandido hasta principios de 2020, momento en que la emergencia sanitaria derivada de la COVID19 impide la asistencia a los centros de estudios. En el caso de la FCEA la definición sobre la modalidad de cursado, horas de clase presencial, trabajo domiciliario, herramientas de evaluación, recursos a utilizar etc. forma parte del contrato pedagógico establecido en las fichas de cada unidad curricular (se denomina unidad curricular a las asignaturas). El proceso de adaptación a la modalidad no presencial implicó un cambio en la ficha que, por necesitar la aprobación de varios actores en distintas 
etapas del proceso, hizo necesario contar con una propuesta de cursada y evaluación en un plazo corto. Esto supuso el desafío de migrar todos los cursos del año 2020 a enseñanza remota a efectos de mantener tanto la cursada como sus correspondientes evaluaciones. Gran parte de este proceso fue posible gracias a la experimentación con diversas modalidades, recursos e instrumentos de evaluación.

En este contexto surgen experiencias de innovación docente, como la de Economía de América Latina, que sientan las bases para el análisis y la reflexión sobre un mejor aprovechamiento de las posibilidades que brindan los formatos mediados por TICs. Esto permite, además, fortalecer la comunidad docente desde el intercambio de buenas prácticas y la reflexión sobre casos particulares y experiencias. El principal objetivo de este trabajo es dejar registro de una experiencia exitosa realizada en este contexto que contribuya a la caja de herramientas de los docentes universitarios de grado, de modo de generar bases sobre las cuales seguir experimentando. En el curso de Economía de América Latina 2020 se instrumentó, como parte de las actividades de evaluación no presencial, la entrega de un trabajo individual a partir de una consigna específica y el diseño e implementación en el EVA de una rúbrica de corrección.

El resto del trabajo incluye 3 secciones. La siguiente sección introduce conceptualmente el uso de herramientas de evaluación como los trabajos individuales y el uso de rúbricas de evaluación. En la sección tercera, se describen las características generales de la asignatura y se presentan las innovaciones didácticas sobre la entrega de tareas, la rúbrica y los resultados encontrados. En la sección cuarta se concluye y se presentan reflexiones para aplicar en próximos cursos, así como sugerencias para otras asignaturas.

\section{Marco teórico}

La educación a distancia debe organizarse de forma que el docente actúe como facilitador del aprendizaje con un rol protagónico del estudiante complementado con evaluación continua de carácter básicamente formativo que permitan hacer devoluciones que guíen el trabajo del estudiante. La elaboración de guías didácticas que recojan los objetivos de aprendizaje, los contenidos a transitar, los recursos y herramientas que acompañarán el curso, no sólo aporta a la formación del estudiante y su aprendizaje autónomo sino también a favorecer el trabajo del docente al hacer explícita la intencionalidad de su labor. Esto genera la necesidad de diseñar instrumentos de evaluación acordes al proceso de enseñanza y aprendizaje que se está desarrollando, que estén alineados a los objetivos del curso, al propósito de la evaluación, a los contenidos y a la población objetivo (Covacevich, 2014).

La evaluación de aprendizajes, independientemente de su modalidad presencial o virtual, debe entenderse como un proceso integrado a la enseñanza como un conjunto de procesos sistemáticos de recogida, análisis e interpretación de información válida y fiable, que en comparación con una referencia o criterio nos permita llegar a una decisión que favorezca la mejora del objeto evaluado (Rodríguez Conde, 2005). La evaluación referida a criterios permite evaluar cuánto los estudiantes han logrado los objetivos propuestos y medir competencias con respecto a un objetivo instruccional, permitiendo al mismo estudiante o a los docentes trabajar en los aspectos identificados como débiles (Covacevich, 2014). En este sentido, se sugirió la implementación de evaluaciones a distancia que cambian el paradigma tradicional de evaluación centrado en la acreditación de conocimientos y recuperación de contenidos, por una modalidad de evaluación continua con énfasis en el carácter formativo y la formación en competencias.

Cada equipo docente se enfrenta al desafío de analizar las características de su curso, sus estudiantes y su equipo docente y sus posibilidades de formarse, generar materiales e instrumentos de evaluación que les permitieran no sólo facilitar el aprendizaje sino también evaluarlo. En algunos casos, el contexto permite evaluaciones sumativas con una mayor frecuencia que en la modalidad presencial a efectos de favorecer la adhesión al curso y generar mejores resultados de aprendizaje a través de la evaluación continua. En cursos con otras características es posible incorporar además instancias de evaluación formativa cuya principal característica es la de facilitar el proceso de aprendizaje sin ser considerada para la evaluación sumativa final (Biggs, 2006, 179). 
En este proceso algunos cursos que tienen como principal característica la masividad, optan por la evaluación automática (Barberá, 2006), recurriendo al armado de grandes bancos de preguntas que reduzcan la posibilidad de fraude y la búsqueda de preguntas que contengan aplicación de contenidos para resolver problemas cuya respuesta pueda sintetizarse en una respuesta numérica, de múltiple opción o emparejamiento. Sin embargo, en los contextos en que la relación entre el número de estudiantes y docentes así lo permitiese se incursiona en evaluaciones de carácter enciclopédico (preguntas de desarrollo o proyectos) y/o colaborativas (foros de discusión y trabajo en grupo incluso interactivo como en el caso de las wikis) en las que concurren habilidades más complejas relacionadas con la selección de información relevante, elaboración personal a partir de la bibliografía recomendada y estrategias metacognitivas entre otros.

Se trata de observar las competencias de un estudiante frente a la tarea de resolver un problema integralmente aplicando sus conocimientos teóricos a situaciones concretas, distintas y cambiantes, esto implica poseer un conjunto de competencias que proceden de su capacitación, de su experiencia y de sus aptitudes personales. En este sentido, la elaboración de instrumentos de evaluación en entornos virtuales de aprendizaje implica de algún modo complementar los procedimientos para evaluar tradicionales, con otros que incorporen metodologías de indagación, investigación formativa y casos prácticos, porque se intenta enlazar en el proceso de evaluación la mejora pedagógica y por lo tanto la evaluación de insumos para el rediseño y mejora de los cursos (Rodríguez-Conde, 2005). Evaluar a distancia, supone diseñar herramientas que permitan discernir el grado de logro de los estudiantes, en un entorno menos controlado, con acceso a materiales y otros recursos, desafiando a evaluar la capacidad de análisis y otras competencias del estudiante en el uso de estos recursos, a diferencia de la evaluación presencial que se puede, potencialmente, concentrar en la recuperación de contenidos.

La comunicación con fines educativos sucede habitualmente en un ambiente físico en el que interactúan varios actores; sin embargo, el contexto de emergencia sanitaria ha trasladado la comunicación fundamentalmente a los entornos virtuales, que permiten la comunicación sincrónica y asincrónica, y el uso de diferentes códigos de lenguaje. Esto hace necesario transparentar los criterios de evaluación a través de consignas claras y exhaustivas. Una consigna es un "conjunto de indicaciones claras y concretas que se organizan en secuencia para que la persona participante o estudiante realice una actividad que es parte de la estrategia de aprendizaje" (Pérez-Chaverri, 2015).

Las consignas deben cumplir determinadas características que faciliten la comunicación clara con el estudiante, entre ellas se cuentan mantener el buen trato, motivar al estudiante, tener un texto escrito claro y comprensible, sin brindar exceso de información y ser factibles de ser realizadas. La consigna deberá explicitar qué hacer y cómo hacerlo en este sentido debe incluir un contexto y fundamentación, la forma de calificación, aspectos formales y plazos de la entrega. El verbo que describe la acción que debe realizar el estudiante y la descripción del producto a entregar son cruciales y es necesario acordar con el estudiante lo que se entiende en cada caso (Atorresi, 2005). Si se pide por ejemplo una reflexión, docente y estudiante deben acordar previamente lo que se espera de esa reflexión. En particular vale la pena especificar en la consigna:

- Bibliografía: se sugiere detallar aquella que es obligatoria o sugerida, así como aclarar si se valorará la incorporación de bibliografía adicional. Por otro lado, es relevante aclarar la modalidad en que se espera que dichos materiales sean referenciados.

- Contenidos: es conveniente aclarar si se trata de una sistematización de información o si se espera un grado de reflexión adicional. Esto último es deseable en tanto se aspire a evaluar la capacidad de análisis del estudiante y a su vez incorporar un componente subjetivo que permita diferenciar los trabajos de uno u otro estudiante de modo de minimizar la posibilidad de fraude. En este sentido también se sugiere la elaboración de consignas alternativas que enfrenta a distintos grupos de estudiantes a problemas diferentes. 
- Aspectos formales: es deseable definir el tipo de archivo a entregar, la longitud, el tipo de letra e interlineado etc. así como también, de ser necesario aspectos como la carátula, índice y bibliografía.

Es fundamental para el estudiante tener claros los aspectos que se van a considerar al momento de evaluar, transparentan el nivel de calidad que debe tener una resolución y pueden mejorar el desempeño estudiantil. Estos criterios pueden incluir aspectos vinculados a la temática y también otros vinculados a aspectos formales. De acuerdo a Anijovich y González (2013) los criterios de evaluación "habilitan formas más ajustadas de aproximación al conocimiento tanto por parte de los alumnos como de los docentes. A través de ellas es posible establecer un diálogo reflexivo [...] explorando los grados de comprensión, los obstáculos y creando oportunidades para pensar el aprendizaje".

La Comisión Sectorial de Enseñanza de la UDELAR recoge varias experiencias de evaluación mediante el uso de rúbricas previas al pasaje a enseñanza remota (Comisión Sectorial de Enseñanza, 2015) en las que se destaca su capacidad de detallar las dimensiones en las que se evaluará el trabajo y los niveles de logro esperados en cada uno de ellos. La rúbrica es una matriz de valoración que incorpora en un eje los criterios o dimensiones a evaluar y en el otro eje una escala de logros. Cada intersección describe explícitamente que se interpreta en cada uno de los niveles de logro de una dimensión. La rúbrica no sólo ayuda a mejorar la consigna agregando aspectos que no estén claros, sino también guía al docente en una corrección ecuánime de los trabajos manteniendo las ponderaciones de las distintas dimensiones y la puntuación de acuerdo a los diferentes niveles de logro.

Algunos autores puntualizan que la dificultad para obtener una buena rúbrica o el tiempo que insume su construcción hacen que no sea un instrumento adecuado. En este sentido, aunque no siempre la primera versión utilizada de una rúbrica es adecuada, suele ser una herramienta que se mejora en la medida que se utiliza y se van detectando omisiones o ajustes necesarios en la definición de los criterios o de los niveles de logro. Por ejemplo, pueden no considerarse todas las dimensiones deseadas o el desarrollo de las descripciones de los niveles de logro de cada una de ellas, que deben centrarse en reducir el margen de subjetividad de los evaluadores, utilizan palabras como "bueno" o "inadecuado" sin especificar qué se entiende por estos conceptos. Sin embargo, en la medida que la rúbrica se utiliza las carencias se van corrigiendo hasta lograr un instrumento de evaluación adecuado.

Otra crítica tiene que ver con la elaboración de la rúbrica "para el estudiante" y no desde el estudiante, esto ha sido resuelto en muchos casos incluyendo al estudiante en el diseño de la rúbrica, esto mejora la comprensión y el uso que el estudiante hace de la misma. Es de uso entregar a los estudiantes la rúbrica conjuntamente con la consigna, esto permite que el estudiante tenga una guía para organizar su trabajo concentrándose en aquellos aspectos que van a ser evaluados, como también deviene en un potente instrumento para una autoevaluación previa a la entrega final.

Las rúbricas pueden ser incorporadas al ambiente de trabajo de moodle, son uno de los mecanismos de calificación avanzada. Moodle permite que los estudiantes vean las rúbricas junto con la consigna actuando como fuera mencionado como instrumento de una primera etapa de autoevaluación. A la vez, facilita la tarea del evaluador en tanto la corrección se realiza por dentro de la plataforma, seleccionando para cada una de las dimensiones el nivel de logro alcanzado por el estudiante. Como resultado, moodle genera además de la calificación final una retroalimentación estandarizada que muestra la descripción del nivel de logro en cada una de las dimensiones y permite agregar comentarios personalizados por parte del evaluador si esto fuera necesario.

En moodle se asigna una calificación numérica a cada uno de los niveles y dimensiones de la rúbrica, esto si bien no es conceptualmente necesario permite obtener de forma simplificada una calificación para la tarea. La puntuación de la rúbrica es la suma de la calificación obtenida en cada una de las dimensiones y se estandariza como porcentaje del puntaje máximo que es posible obtener. El evaluador puede elegir diferentes escalas, mínimas y máximas para cada una de ellas. Si bien la rúbrica en moodle está pensada 
para la evaluación sumativa y por lo tanto requiere el uso de calificaciones numéricas en cada dimensión, estas pueden ser ocultadas al estudiante de modo de utilizar la rúbrica para realizar evaluación formativa en la que el estudiante sólo acceda a la retroalimentación (Moodle, 2019).

En la siguiente sección se presenta como caso de estudio, la aplicación de la rúbrica para la evaluación de trabajos entregados por estudiantes avanzados de la asignatura de Economía de América Latina de la Licenciatura en Economía de la UDELAR.

\section{Caso de estudio}

\subsection{Descripción de la asignatura Economía de América Latina}

Economía de América Latina integra la Unidad Académica de Historia y Desarrollo y corresponde al 5to semestre de la carrera de la Licenciatura en Economía de la Facultad de Ciencias Económicas y de Administración. Además, se ofrece como opcional a otras carreras de ciencias sociales interdisciplinarias (Licenciaturas en Sociología, Desarrollo, Ciencia Política, Relaciones Internacionales, etc.). La Licenciatura en Economía está conformada por 8 semestres (4 años), un total de 360 créditos y se organiza en cursos obligatorios y optativos, con un mínimo de créditos por áreas de conocimiento. Se trata de una asignatura obligatoria que deben tomar los estudiantes, equivale a 10 créditos y está pensado sobre la base de que el alumno cuente con conocimientos teórico-metodológicos básicos previos de la economía y/u otras disciplinas sociales. La matrícula por curso es en promedio 297 estudiantes (promedio de inscriptos entre 2017 y 2020).

El objetivo de la asignatura es brindar un panorama del pensamiento latinoamericano sobre cuestiones del desarrollo económico y de los distintos modelos de desarrollo por los que pasó la región en sus distintas etapas históricas. Además, propone reflexionar sobre los principales desafíos y problemas de América Latina desde la independencia hasta nuestros días. El desarrollo del curso toma como eje central las bases del pensamiento latinoamericano sobre desarrollo económico: el estructuralismo y el concepto de dependencia. Está organizado en 5 módulos temáticos que siguen un orden cronológico: i) crecimiento hacia afuera y conformación de las economías primario-exportadoras; ii) crecimiento hacia dentro y las fases de la industrialización; iii) la reestructuración económica desde la década de 1980; iv) los debates sobre la evaluación de las reformas y las propuestas críticas; v) el contexto internacional del siglo XXI y los debates actuales sobre los recursos naturales, crecimiento y distribución y la nueva geopolítica. Los 4 primeros módulos constituyen el núcleo duro del curso, mientras que el 5to módulo aspira a introducir temas de debate de la actualidad e investigación reciente sobre estos temas. El curso propone una bibliografía obligatoria bastante extensa, que prioriza los aportes de la literatura latinoamericana sobre los temas.

En cuanto a la modalidad de enseñanza, el curso se imparte tradicionalmente bajo la modalidad de teóricos y seminarios (tipo taller), con dos clases semanales de 2 horas. En los teóricos, las instancias son más que nada clases magistrales a cargo de los profesores encargados, con participación y ejercicios genéricos. En los seminarios predomina el ejercicio práctico y de discusión en grupos más reducidos a cargo de un profesor ayudante.

El sistema de evaluación del curso reglamentado que se dictaba hasta el 2019, incluía 2 parciales escritos presenciales (40 puntos cada uno); la participación oral, materiales escritos preparados durante el curso y controles de lectura (20 puntos). Para la aprobación de la materia se exigía como requisito un mínimo de $40 \%$ en cada parcial y $40 \%$ en la participación. En total, el mínimo debe superar el $50 \%$ de los puntos.

La modalidad virtual adoptada a raíz de la pandemia, introduce cambios en el sistema de evaluación que se transforma totalmente a distancia y se realiza a través de diversas actividades que comprenden pruebas 
individuales y participación en grupos prácticos. El requisito mínimo para la exoneración del curso es 50 puntos (sobre un total de 100) y se elimina la exigencia de puntos mínimos por pruebas. Las evaluaciones individuales (suman 70 puntos en total) incluyeron dos actividades. Un cuestionario (30 puntos) a través del EVA en un día y horario predeterminado, que incluyó dos partes: cuestionario abierto de preguntas (múltiple opción y verdadero-falso) y un set de preguntas abiertas. La segunda prueba consiste en la realización de un trabajo individual (70 puntos), a entregar en el EVA luego de finalizar el curso, cuyo objetivo es evaluar los contenidos trabajados en la segunda parte del curso. Sobre esta actividad nos extenderemos en la siguiente sección.

En relación con la participación en los talleres (30 puntos), la asignación de puntos se distribuye entre siete actividades cuyo principal objetivo es incentivar la participación activa y continua de los estudiantes. Esto incluye: dos controles de lectura en el EVA uno individual y uno grupal, cuatro entregas de trabajos grupales, con consignas y plazos bien definidos y la participación individual en las instancias sincrónicas (clases virtuales) o a través de los foros.

\subsection{Descripción de la entrega de tareas y de la rúbrica}

Esta sección se centra en la entrega del trabajo final, y la rúbrica creada para su correspondiente evaluación. Esta prueba tiene como propósito evaluar la comprensión de los temas recorridos en la segunda mitad del curso. A lo largo de las diferentes instancias, los estudiantes realizaron trabajos siguiendo consignas con preguntas guiadas en base a lecturas previamente asignadas. Siguiendo el mismo tipo de consigna, con la que los estudiantes están familiarizados, en este trabajo final, individual, se presenta una serie de preguntas de desarrollo a ser elaboradas en base a los textos asignados. Se espera que los estudiantes logren satisfactoriamente comprender los temas planteados, sistematicen de forma adecuada, logren interpretar y realizar conexiones entre los diferentes textos, y por último desarrollen reflexiones personales.

Se trata de un trabajo de creación individual. Si bien los estudiantes pueden intercambiar con sus compañeros ideas, reflexiones, dudas, la realización y redacción del trabajo es individual. Además, se espera que los estudiantes hagan un uso adecuado de las referencias bibliográficas y los materiales que utilicen (sitios web, videos, etc.). En todos los casos deben citar correctamente las fuentes utilizadas en el trabajo. Se les proporciona un material de apoyo para guiarlos. En caso de dudas con el uso de las referencias se anima a consultar a los docentes. Se dispone de mecanismos para el control de comportamiento poco éticos como pueden ser las conductas colaborativas y/o situaciones de plagio. Al momento de la corrección, en caso de dudas sobre el comportamiento ético de los estudiantes se anula el trabajo y según el caso, se realiza una evaluación oral a los estudiantes involucrados. Por otro lado, se presentan claramente los aspectos formales del trabajo, como fecha de entrega, forma de entrega, extensión (tipo de letra, cantidad de carillas), proporción de datos personales, tipo de archivo a entregar, instancias de retroalimentación en base al uso de la rúbrica de evaluación.

Por último, gran parte del esfuerzo estuvo centrado en la creación de la rúbrica de evaluación. Con esta rúbrica se detallan los aspectos que se evaluarán en cada una de las preguntas planteadas en la consigna y lo que se espera de cada ítem (Ver Tabla 1). El puntaje máximo a alcanzar es de 40 puntos. La tabla 1 muestra los 4 criterios generales a ser evaluados: i) capacidad de sistematización de determinados temas, ii) capacidad de análisis de los textos y aplicación de conceptos, así como comparación entre diferentes textos o el uso de ejemplos, iii) capacidad de reflexión, discusión y evaluación, y iv) redacción y coherencia del trabajo. Es importante aclarar que la valoración personal debe ser adecuada a un nivel universitario, es decir con sustentación conceptual o que refleje los conceptos manejados en el curso. En base a estos criterios se define cuando el estudiante alcanza una nota de excelente, bueno, y aceptable. Una respuesta insuficiente es aquella que no cumple con la consigna, no responde la pregunta, responde la pregunta equivocadamente, toda la respuesta es un error, carece de referencias bibliográficas o el trabajo en su totalidad es difícil de leer y contiene errores ortográficos y gramaticales.

En cuanto a los puntajes, el trabajo individual se divide en 3 preguntas que contiene 3 partes (a, b y c). 
Cada una de estas preguntas tiene el mismo puntaje (12 puntos) que sumadas dan 36 puntos y los restantes 4 puntos corresponden a la redacción del texto en su conjunto, que incluye gramática, ortografía, sintaxis, citas y bibliografía (Tabla 1). Cada pregunta vale 12 puntos y cada subparte de la pregunta (a, b y c) vale 4.

\begin{tabular}{|c|c|c|c|}
\hline & EXCELENTE & BUENO & ACEPTABLE \\
\hline $\begin{array}{l}\text { Parte a) dentro de cada } \\
\text { pregunta: } \\
\text { Sistematización de } \\
\text { determinados temas en } \\
\text { los textos indicados }\end{array}$ & $\begin{array}{l}\text { La sistematización incluye los } \\
\text { aspectos relevantes del tema. Se } \\
\text { identifica el argumento principal } \\
\text { y la manera que usan los autores } \\
\text { para validarlo }\end{array}$ & $\begin{array}{l}\text { La sistematización } \\
\text { incluye los aspectos } \\
\text { relevantes del tema. } \\
\text { Presenta algún error } \\
\text { tanto para } \\
\text { identificar el } \\
\text { argumento principal } \\
\text { como la manera que } \\
\text { usan los autores } \\
\text { para validarlo }\end{array}$ & $\begin{array}{l}\text { La sistematización no } \\
\text { incluye los aspectos } \\
\text { relevantes del tema. } \\
\text { Presenta más de dos } \\
\text { errores tanto para } \\
\text { identificar el argumento } \\
\text { principal así como la } \\
\text { manera que usan los } \\
\text { autores para validarlo }\end{array}$ \\
\hline $\begin{array}{l}\text { Parte b) dentro de cada } \\
\text { pregunta: Análisis del } \\
\text { texto y aplicación de } \\
\text { conceptos/ comparación } \\
\text { entre diferentes } \\
\text { textos/Ejemplificaciones }\end{array}$ & $\begin{array}{l}\text { El análisis hace dialogar a todos } \\
\text { los textos/ Interpreta } \\
\text { correctamente los conceptos } \\
\text { propuestos sin errores/Selecciona } \\
\text { y justifica adecuadamente un } \\
\text { ejemplo interesante }\end{array}$ & $\begin{array}{l}\text { El análisis hace } \\
\text { dialogar a casi todos } \\
\text { los textos, pero no a } \\
\text { todos/ Interpreta } \\
\text { bien los conceptos } \\
\text { pero falta mayor } \\
\text { vínculo con los } \\
\text { aspectos teóricos / } \\
\text { Selecciona un } \\
\text { ejemplo interesante } \\
\text { pero no lo justifica } \\
\text { de forma completa }\end{array}$ & $\begin{array}{l}\text { El análisis no hace } \\
\text { dialogar a todos los } \\
\text { textos de forma } \\
\text { adecuada/ Interpreta los } \\
\text { conceptos con más de } \\
\text { dos errores conceptuales } \\
\text { y falta mayor vínculo } \\
\text { con los aspectos teóricos } \\
\text { /Selecciona un ejemplo } \\
\text { satisfactorio pero no lo } \\
\text { justifica de forma } \\
\text { completa }\end{array}$ \\
\hline $\begin{array}{l}\text { Parte c) dentro de cada } \\
\text { pregunta: } \\
\text { Reflexión/discusión/ } \\
\text { evaluación }\end{array}$ & $\begin{array}{l}\text { Las reflexiones incorporan } \\
\text { aspectos conceptuales trabajados } \\
\text { en el curso y revisados en los } \\
\text { textos, es clara y sustancial, } \\
\text { aporta una valoración personal }\end{array}$ & $\begin{array}{l}\text { Las reflexiones } \\
\text { incorporan aspectos } \\
\text { conceptuales } \\
\text { trabajados en el } \\
\text { curso y revisados en } \\
\text { los textos, pero no } \\
\text { es suficiente la } \\
\text { valoración personal }\end{array}$ & $\begin{array}{l}\text { Las reflexiones } \\
\text { incorporan aspectos } \\
\text { conceptuales trabajados } \\
\text { en el curso y revisados } \\
\text { en los textos, sin } \\
\text { valoración personal }\end{array}$ \\
\hline $\begin{array}{l}\text { Redacción y coherencia } \\
\text { del trabajo }\end{array}$ & $\begin{array}{l}\text { Los argumentos presentados } \\
\text { tienen un orden lógico. Es un } \\
\text { trabajo fácil de leer, bien } \\
\text { redactado y sin errores } \\
\text { gramaticales ni ortográficos. } \\
\text { Fuentes referidas y citadas } \\
\text { correctamente. } \\
\text { Presenta correctamente la } \\
\text { bibliografía, incluyendo a todos } \\
\text { los materiales que se han } \\
\text { referenciado en el texto. }\end{array}$ & $\begin{array}{l}\text { Algunos } \\
\text { argumentos } \\
\text { presentados no } \\
\text { tienen un orden } \\
\text { lógico. El trabajo se } \\
\text { lee con pocas } \\
\text { dificultades. Pocos } \\
\text { errores gramaticales } \\
\text { y ortográficos. } \\
\text { Fuentes mal citadas } \\
\text { o referenciadas. } \\
\text { Incluye bibliografía. }\end{array}$ & $\begin{array}{l}\text { Muchos argumentos no } \\
\text { tienen orden lógico. El } \\
\text { trabajo no es fácil de } \\
\text { leer. Muchos errores } \\
\text { gramaticales y } \\
\text { ortográficos. Las fuentes } \\
\text { no están citadas. Incluye } \\
\text { bibliografía, aunque } \\
\text { incorrecta. }\end{array}$ \\
\hline
\end{tabular}

Fuente: Elaboración propia

Tabla 1: Rúbrica de evaluación 


\subsection{Resultados sobre el trabajo individual y la rúbrica}

En relación con los resultados del trabajo individual, un primer análisis tiene que ver con los puntajes obtenidos por los estudiantes. En este caso, dado que no se establecieron puntos mínimos, ningún estudiante fue descalificado por su puntaje final. La nota promedio fue de 29 en un total de 40 puntos, y solamente 14 trabajos en un total de 216 tuvieron una calificación menor a 20 puntos. Estos resultados son mejores que los alcanzados en la evaluación tradicional realizada a través una prueba presencial escrita individual.

La calidad de los trabajos escritos fue acorde a lo esperado. Dado que era la primera vez que se establecía este formato de evaluación (domiciliario), la preocupación de los docentes estaba concentrada en que los estudiantes lograran realizar un trabajo auténtico, sin plagios ni copias entre ellos. Se realizaron instancias sincrónicas de intercambio dedicadas específicamente a explicar la consigna escrita del trabajo, así como también se evacuaron todas las dudas surgidas por mails o por el foro en la plataforma. Este seguimiento fue parte de un esfuerzo conjunto. La entrega de la rúbrica con antelación les permitía a los estudiantes conocer los criterios de corrección y un rango de la calificación final que podrían obtener. No se detectaron problemas éticos en la realización de la tarea propuesta.

Para conocer la opinión de los estudiantes, al finalizar el curso se realizó un cuestionario anónimo, a completar online de forma voluntaria, en donde se preguntó por diversos aspectos del curso. El cuestionario fue respondido por 122 estudiantes (una tasa de respuesta del $40 \%$ en relación con el total estudiantes inscriptos a la asignatura). Una de las preguntas aspiraba a relevar el punto de vista sobre la herramienta de evaluación aplicada en la segunda parte del curso: “¿Cómo evalúa la propuesta de la segunda revisión en base a un trabajo individual a entregar?", en una escala de 1 a 5 (siendo 1 muy malo y 5 excelente). Los resultados a esta pregunta, un $30 \%$ calificó de 4 y un $44 \%$ de 5 a dicha propuesta (Gráfico 1). Estas respuestas de los estudiantes demuestran una valoración muy favorable a la propuesta de trabajo individual y su formato de evaluación.

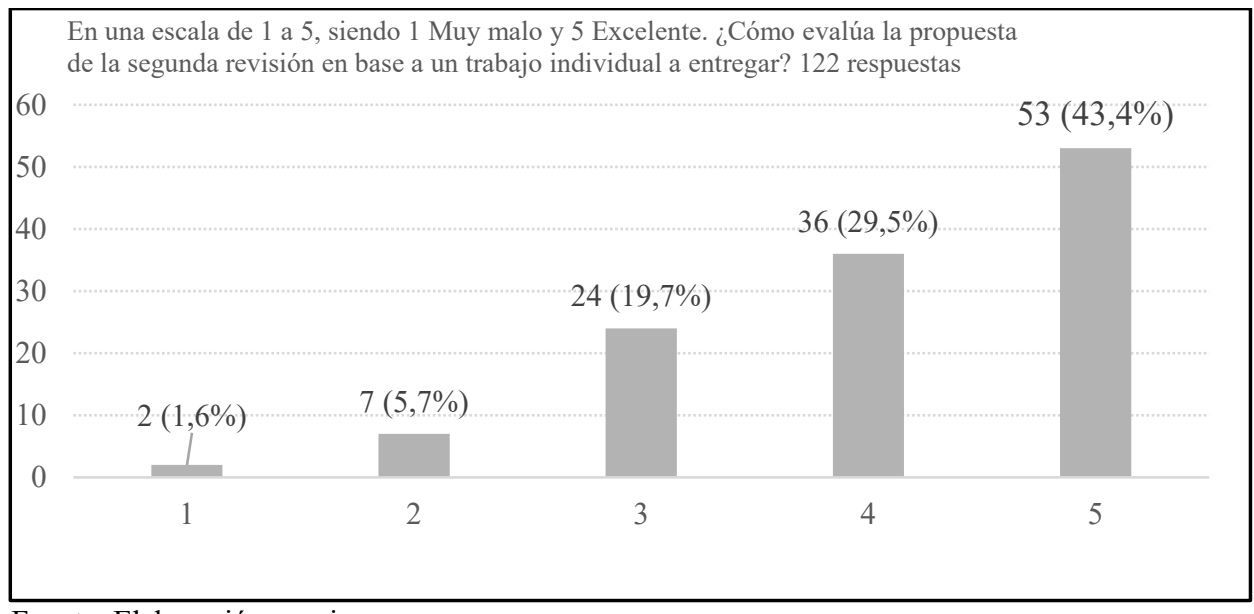

Fuente: Elaboración propia

Gráfico 1: Respuestas de los estudiantes sobre el trabajo individual

Además de la valoración cuantitativa, al final del cuestionario, se dio espacio para que los estudiantes escribieran sugerencias y/o comentarios del curso (de libre redacción). En relación al trabajo individual, uno de los problemas que algunos estudiantes declararon haber tenido es con la restricción de la cantidad de páginas establecidas (la consigna establecía no más de 3 carillas por pregunta, en total 9 carillas). De todas maneras, es de destacar que se buscó específicamente que las respuestas fueran concisas y cortas para fomentar la selección y sistematización de la información y evitar el resumen de los textos. Por otro lado, otros estudiantes tuvieron dificultades para poder cumplir con los plazos asignados, y si bien la consigna fue entregada con un mes de antelación, algunos manifiestan que fue insuficiente. Al respecto, 
cabe mencionar que la entrega de este trabajo coincide con la realización de pruebas de otras asignaturas que están cursando los estudiantes.

\section{Conclusiones}

El contexto causado por la pandemia otorgó una oportunidad para experimentar con nuevas herramientas de aprendizaje en entornos virtuales. Esto significó un importante desafío tanto para los docentes como para los estudiantes, no acostumbrados a explotar todas las potencialidades que las TICs permiten. Además de tener que aprender nuevas herramientas, fue necesario que esto sucediera de forma rápida. Esto implicó, un proceso de ensayo, de prueba y error limitado en el tiempo.

Se utilizó la entrega de un trabajo individual, domiciliario y el diseño de una rúbrica para su corrección, todo implementado a través del entorno virtual de aprendizaje. Esta innovación en el proceso de evaluación del aprendizaje fue valorada muy positivamente, tanto por los docentes como por los estudiantes. Una ventaja relevante del uso de la rúbrica, es transmitir con claridad a los estudiantes lo que se espera del producto a entregar, y con este criterio, los docentes puedan corregir los trabajos. Al tratarse de la primera vez que se aplicó en esta asignatura, quedan en la agenda opciones sobre su uso para experimentar en próximas ediciones y/o en otras asignaturas. En este caso, se optó por proponer la misma consigna a todos los estudiantes, y otra alternativa sería proponer consignas diferentes por grupos de estudiantes. En este caso, en el juicio general, se dejó un margen de puntaje máximo para los estudiantes que incorporan nueva bibliografía e incorporan ejemplos. En este aspecto, es necesario profundizar las acciones que incentiven la búsqueda de bibliografía adicional. Por último, se considera la posibilidad de mejorar una de las preguntas cuyo objetivo era generar la reflexión individual a través del trabajo previo con los estudiantes, dando mayor cantidad de ejemplos que ellos tomen como referencia.

\section{Referencias}

Anijovich, R., González, C. (2013) Evaluar para aprender. Conceptos e instrumentos. Aique Grupo Editor.

Atorresi, A. (2005) Taller de escritura II. Las respuestas a consignas de escritura académica. Material de Posgrado en Constructivismo y Educación, Buenos Aires, FLACSO-Argentina y UAM.

Barberá, E. (2006) Aportaciones de la tecnología a la e-evaluación. Revista De Educación a Distancia (RED), Monográfico VI (2006): Evaluación en entornos virtuales de aprendizaje, pp.1-13.

Biggs, J. (2006) Calidad del aprendizaje universitario. 2a. Madrid: Narcea ediciones.

Covacevich, C. (2014) Cómo seleccionar un instrumento para evaluar aprendizajes estudiantiles. División de Educación (SCL/EDU), Nota Técnica, IDB-TN-738, Banco Interamericano de Desarrollo, Diciembre.

Comisión Sectorial de Enseñanza. Unidad Académica (2015) La evaluación en la educación superior: un escenario de controversia, Temas de enseñanza, n. ${ }^{\circ}$ 2, Montevideo: Universidad de la República, Comisión Sectorial de Enseñanza.

Moodle (2019) Rúbricas en Moodle. Documentación de moodle. Disponible en:

https://docs.moodle.org/all/es/P\%C3\%A1gina Principal (fecha consulta: 12 octubre de 2020)

Pérez-Chaverri, J. (2015) Conceptualizando las consignas como parte de las actividades de un curso virtual: una experiencia basada en los procesos de capacitación en la UNED. Revista electrónica calidad en la educación superior, 6(2), pp. 136-163.

Rodríguez-Conde, M.J. (2005) Aplicación de las TIC a la evaluación de alumnos universitarios. Teoría de la Educación. Educación y Cultura en la Sociedad de la Información, 6(2), pp. 1-17. 